\title{
Pseudoglandular Formation in Hepatocellular Carcinoma Determines Apparent Diffusion Coefficient in Diffusion-Weighted MRI
}

\author{
In Kyung Park, Jeong-Sik Yu, Eun-Suk Cho, Joo Hee Kim, Jae-Joon Chung \\ Department of Radiology, Gangnam Severance Hospital, Yonsei University College of Medicine, \\ Seoul, Korea
}

\section{Original Article}

Received: February 26, 2018

Revised: March 23, 2018

Accepted: March 27, 2018

\section{Correspondence to:}

Jeong-Sik Yu, M.D.

Department of Radiology,

Gangnam Severance Hospital,

Yonsei University College

of Medicine, 211 Eonju-ro,

Gangnam-gu, Seoul 06273,

Korea.

Tel. +82-2-2019-3510

Fax. +82-2-3462-5472

E-mail: yjsrad97@yuhs.ac

This is an Open Access article distributed under the terms of the Creative Commons Attribution Non-Commercial License (http://creativecommons.org/licenses/ by-nc/3.0/) which permits unrestricted non-commercial use, distribution, and reproduction in any medium, provided the original work is properly cited.

Copyright (C) 2018 Korean Society of Magnetic Resonance in Medicine (KSMRM)

Purpose: To determine the impact of pseudoglandular formation on apparent diffusion coefficient (ADC) values of hepatocellular carcinoma (HCC) in diffusionweighted imaging (DWI), and to validate the results using histopathological grades.

Materials and Methods: We assessed 182 HCCs surgically resected from 169 consecutive patients. Each type of tumor pseudoglandular formation was categorized into "non-," "mixed-," or "pure-," based on official histopathology reports. The ADC for each tumor was independently measured, using the largest region of interest on the ADC map. Data were assessed using the analysis of variance test, with Bonferroni correction for post hoc analysis to stratify the relationship of ADCs with pseudoglandular formation, followed by subgroup analysis according to the histopathological tumor grades.

Results: The mean ADC was significantly higher in pure pseudoglandular lesions ( $\mathrm{n}$ $\left.=5,1.29 \pm 0.08 \times 10^{-3} \mathrm{~mm}^{2} / \mathrm{s}\right)$ than in non-pseudoglandular lesions $(\mathrm{n}=132,1.08$ $\left.\pm 0.17 \times 10^{-3} \mathrm{~mm}^{2} / \mathrm{s} ; \mathrm{P}=0.003\right)$ or mixed-pseudoglandular lesions $(\mathrm{n}=45,1.16 \pm$ $0.24 \times 10^{-3} \mathrm{~mm}^{2} / \mathrm{s} ; \mathrm{P}=0.034$ ). The $A D C$ values and pseudoglandular formation were significantly correlated in moderately differentiated HCCs $(n=103 ; r=0.307, P=$ $0.007)$, while well- $(n=19)$ and poorly-differentiated HCCs $(n=60)$ did not show significant correlation $(r=0.105$ and 0.068 , respectively; $P=0.600$ and 0.685 , respectively).

Conclusion: The degree of pseudoglandular formation could be one of the determinants of ADC in DWI of HCCs-especially moderately differentiated HCCs-while its influence does not appear to be significant in well- or poorly differentiated HCCs.

Keywords: Diffusion magnetic resonance imaging; Carcinoma, hepatocellular; Liver diseases; Liver neoplasms

\section{INTRODUCTION}

Hepatocellular carcinoma (HCC) is a commonly diagnosed malignancy and one of the main causes of cancer-related deaths worldwide, especially in Asia $(1,2)$. A variety of methods have been suggested to predict the prognosis of HCC via imaging, in order to facilitate diagnosis and treatment planning. Notably, magnetic resonance imaging (MRI) has been effectively used in preoperative evaluation. Diffusion-weighted imaging (DWI) 
is used to characterize internal structures based on the diffusion properties of water molecules in tissues, reflected by the apparent diffusion coefficients (ADCs) $(3,4)$. It is included in a routine protocol for diagnostic liver MRI during the assessment of focal lesions (5-8). Recently, several studies have reported that ADCs in DWI serve to reliably stratify HCCs into different histological grades because of their negative correlation with tumor grades (9-16).

$A D C s$ are assumed to be determined by several factors, such as the volume of extracellular space, which is related to the size of the tumor cells (represented by histopathological tumor grade), viscosity, and/or interstitial fibrosis, in addition to capillary perfusion. The cellular architecture in the tumor may also affect ADCs, for example, glandular formation results in increased motion of the water molecules, thereby influencing ADC values (17, 18). In daily clinical practice, HCCs with high ADC values have been encountered rather frequently; some of these lesions were surgically confirmed to exhibit a large amount of pseudoglandular formation. To our knowledge, there has been no scientific report published on this topic, involving a large population. Thus, this study sought to validate the previously reported relationship between the $A D C$ values of HCCs and pseudoglandular formation in the tumors, followed by comparative validation using histopathological tumor grades.

\section{MATERIALS AND METHODS}

\section{Subjects}

The Institutional Review Board of our institution (Gangnam Severance Hospital, Seoul, Republic of Korea) approved this retrospective study and waived the requirement for informed consent. Eligible patients were identified by querying the database of all patients who were surgically diagnosed with HCC at our institution between January 2011 and December 2017, and 363 potential candidates were selected.

Our analysis included the following: patients 1) who had undergone preoperative liver MRI (including dynamic enhanced imaging and DWI) within 2 months prior to surgery; 2 ) whose location of tumor on MRI was identical to the location of the surgically resected HCC in the pathology report; 3) whose pathology report described the histopathological grade based on the Edmonson and Steiner grading system (I-IV) and architectural patterns (trabecular, pseudoglandular, or compact pattern); and
4) whose definitive localization of the lesion on DWI facilitated determination of the regions of interest (ROIs). Patients whose lesion size was $<1 \mathrm{~cm}$ (to avoid the partial volume averaging effect) or whose tumor was treated using transcatheter arterial chemoembolization or radiofrequency ablation before imaging were excluded from the analysis. A total of 182 lesions from 169 patients (range, 38-81 years; mean age, 58.3 years; male: female ratio of 134:35) were finally included in the study. Most patients exhibited underlying liver disease: chronic hepatitis $B(n=140)$, hepatitis $C(n=10)$, hepatitis $B$ and $C(n=1)$, or alcoholic cirrhosis $(n=2)$ while the remaining 16 patients had no known underlying liver disease.

\section{MRI Protocol}

All MRI examinations were performed with a 1.5-T MRI system (Magnetom Avanto, Siemens, Erlangen, Germany) that was equipped with high-performance gradients (maximum amplitude $45 \mathrm{mT} / \mathrm{m}$ ) and a six-element phasedarray surface coil. Before dynamic MRI, the non-contrast fast T2- and T1-weighted MRI using half-Fourier singleshot turbo-spin-echo (HASTE, repetition time [TR] infinite, echo time [TE] $84 \mathrm{~ms}$, flip angle $150^{\circ}$, echo train length 256 , slice thickness $6 \mathrm{~mm}$, slices 27) and double-echo chemical shift gradient echo sequences (TR $100 \mathrm{~ms}$, first-echo TE $2.0 \mathrm{~ms}$ [opposed phase], second-echo TE $4.2 \mathrm{~ms}$ [in-phase], flip angle $70^{\circ}$ ) were obtained. A fat-suppressed threedimensional (3D) gradient echo sequence (i.e., volumetric interpolated breath-hold examination [VIBE]; Siemens, Erlangen, Germany) was used for multiphasic dynamic imaging via intravenous gadoxetic acid injection (Primovist, Bayer Schering, Berlin, Germany; $0.025 \mathrm{mmol} / \mathrm{kg}$ ).

Respiratory-triggered DWI with single-shot echo planar imaging was obtained with the following scanning parameters: two $b$ factors, $50 \mathrm{~s} / \mathrm{mm}^{2}$ and $800 \mathrm{~s} / \mathrm{mm}^{2} ; \mathrm{TR}$, $3900 \mathrm{~ms} ; \mathrm{TE}, 75 \mathrm{~ms}$; matrix size, $156 \times 192$; average, 6; 54-60 slices (27-30 for each $b$ factor); slice thickness, 6 $\mathrm{mm}$; and interslice gap, 20\%. The ADC values for each DWI sequence were automatically calculated by the MRI system, and the corresponding ADC maps were created. The last sequence, the delayed hepatobiliary phase, was acquired with the VIBE sequence, 20 min after contrast media injection.

\section{Data Analysis}

All patients' images, pathology reports, and medical records were preliminarily reviewed by the study coordinator (a radiologist with 20 years of experience in abdominal 
MRI), who marked an arrow on each HCC on the gadoxetic acid-enhanced hepatobiliary phase images. Two radiologists (a radiologist with 10 years of experience in abdominal MRI and a fourth-year resident), who were blinded to the histopathological grade of the lesions, independently drew an $\mathrm{ROI}$ on the ADC map to measure the ADC value of each tumor. The largest ROI was placed on the enhancing or non-necrotic solid portion in each tumor by referring to dynamic imaging and other sequence images, such as the pre-contrast T1- and T2-weighted images and DWI. To minimize the partial volume average affect, each tumor margin was excluded. In the absence of clear demarcation of the lesion on the ADC map because of its small size, we simultaneously displayed the corresponding DWI images (b value, $50 \mathrm{~s} / \mathrm{mm}^{2}$ ) on picture archiving and communication system monitors. To define the $x$ and $y$ coordinates of the lesion on the DWI image, we drew two horizontal and perpendicular lines passing through the center of the lesion from the left border and the upper border of the image; two identical lines were drawn on the corresponding ADC map (19). The manually synchronized center of the lesion was located and the ROI was placed to measure the ADC values. The average values obtained by the two radiologists were used for statistical analyses.

The pathologists at our institution reported both the major and worst Edmonson and Steiner grades of all surgically resected HCCs. In the present study, the major grade was considered to represent the imaging characteristics of each lesion and was used for analyses. All HCC lesions were categorized into three groups according to their grades: 19 well differentiated (wd) Edmonson and Steiner grade 1 lesions; 103 moderately differentiated $(\mathrm{md})$ grade 2 lesions; and 60 poorly differentiated (pd) HCCs including grade 3 ( $=52)$ and grade $4(n=8)$ lesions. Based on the architectural patterns of HCCs focused on the pseudoglandular (PG) type, the lesions were divided into three groups according to the

Table 1. The Number of Each Subgroup of Pseudoglandular Types and Tumor Grades of Hepatocellular Carcinomas

\begin{tabular}{|c|c|c|c|c|}
\hline & \multicolumn{3}{|c|}{ Pure-PG HCCs Mixed-PG HCCs Non-PG HCCs } & Total \\
\hline wd-HCCs & 1 & 2 & 16 & 19 \\
\hline $\mathrm{md}-\mathrm{HCCs}$ & 3 & 31 & 69 & 103 \\
\hline $\mathrm{pd}-\mathrm{HCCs}$ & 1 & 12 & 47 & 60 \\
\hline Total & 5 & 45 & 132 & 182 \\
\hline
\end{tabular}

pathology reports: five pure-PGs, 45 mixed-PGs and 132 non-PGs (Table 1).

\section{Statistical Analysis}

The inter-observer agreement of ADC measurement between the two observers was examined using the BlandAltman test. Following the Kolmogorov-Smirnov test of the data distribution pattern of the measured ADC values, an analysis of variance (ANOVA) test was performed to verify the relationships between the mean ADCs and two factors (i.e., the histopathological grades and the architectural patterns). It was followed by multivariate regression analysis to confirm the independent effect of each factor. To stratify the relationship of ADCs in each subgroup of histopathological tumor grade based on architectural pattern and vice versa, ANOVA with Bonferroni correction for post hoc analysis was performed. All statistical analyses were performed using MedCalc (MedCalc Software bvba, Ostend, Belgium, version 17.9), and statistical significance was set at $\mathrm{P}<0.05$.

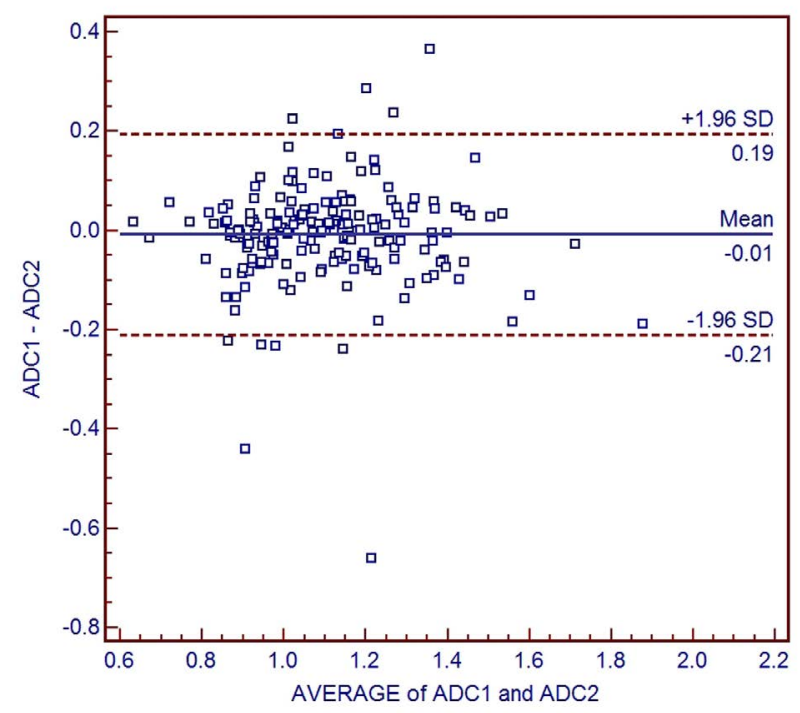

Fig. 1. Bland-Altman plot comparing interobserver agreement of apparent diffusion coefficients (ADCs). The blue line represents the absolute difference, while the two dotted red lines represent the $95 \%$ confidence interval of the mean difference. The mean absolute difference in the ADC measurements between the two observers is 0.010 $\times 10^{-3} \mathrm{~mm}^{2} / \mathrm{s}$ (limit of agreement, 0.20-0.66). ADC1 and 2 indicate ADCs measured by observers 1 and $2\left(\times 10^{-3} \mathrm{~mm}^{2} / \mathrm{s}\right)$, respectively. 


\section{RESULTS}

The Bland-Altman plot (Fig. 1) revealed that there was no fixed or proportional bias in ADC assessment, and substantial reproducibility of the ADC measurement between the two observers was confirmed. The mean absolute difference in the $A D C$ measurement between the two observers was $0.010 \times 10^{-3} \mathrm{~mm}^{2} / \mathrm{s}$ (limit of agreement, $0.20-0.66 \times 10^{-3} \mathrm{~mm}^{2} / \mathrm{s}$ ). The overall ADC data exhibited sufficient normality to fit subsequent statistical analyses $(P$
$>0.05)$.

For the pseudoglandular pattern, the mean ADC of pure$P G$ types $\left(1.29 \pm 0.08 \times 10^{-3} \mathrm{~mm}^{2} / \mathrm{s}\right)$ was significantly higher than the mean ADC of mixed-PG types $\left(1.16 \pm 0.24 \times 10^{-3}\right.$ $\left.\mathrm{mm}^{2} / \mathrm{s}\right)$ or non-PG types $\left(1.08 \pm 0.17 \times 10^{-3} \mathrm{~mm}^{2} / \mathrm{s}\right)$ (Figs. 2, 3a). The three groups showed different overall values $(P=$ 0.003). When comparing the mean ADCs between two of the three pseudoglandular patterns, significant differences were detected (pure-PG vs. mixed-PG, $P=0.034$; pure-PG vs. non-PG, $P=0.003$; mixed-PG vs. non- $P G, P=0.030$ ).

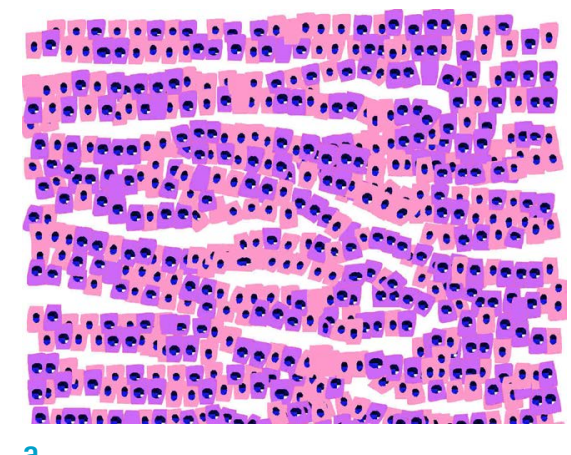

a
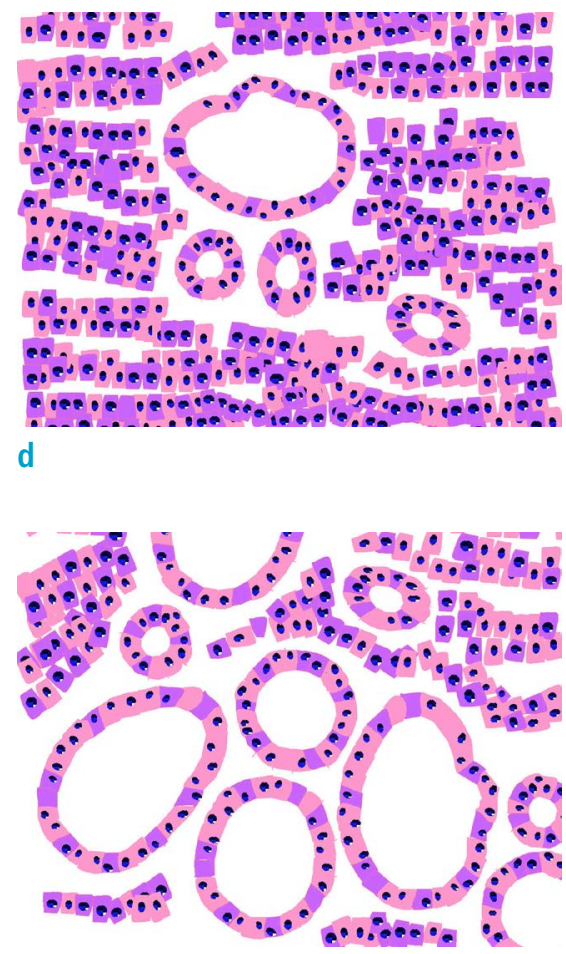

g

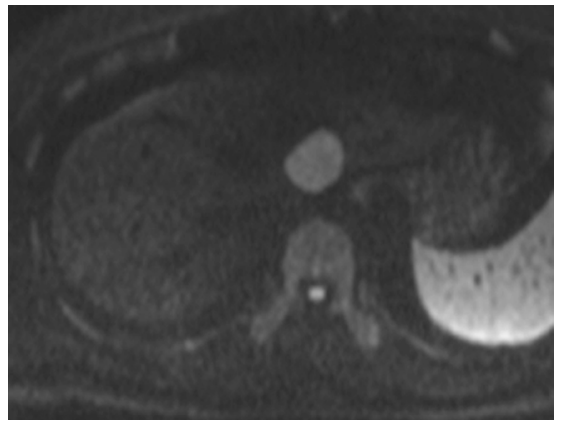

b

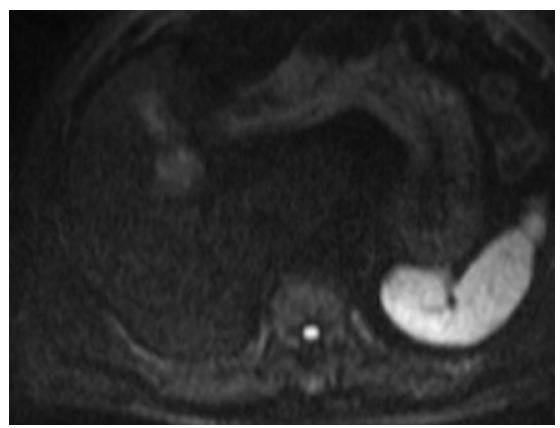

e

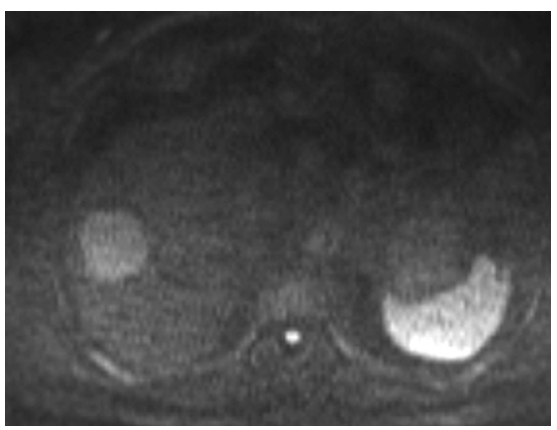

h

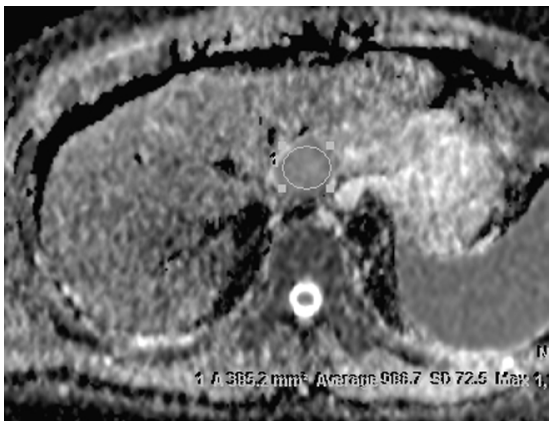

c

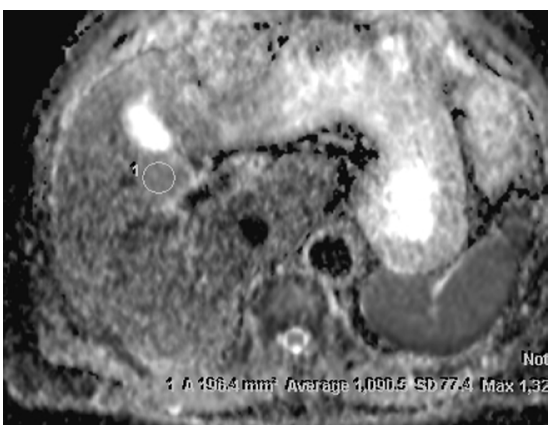

f

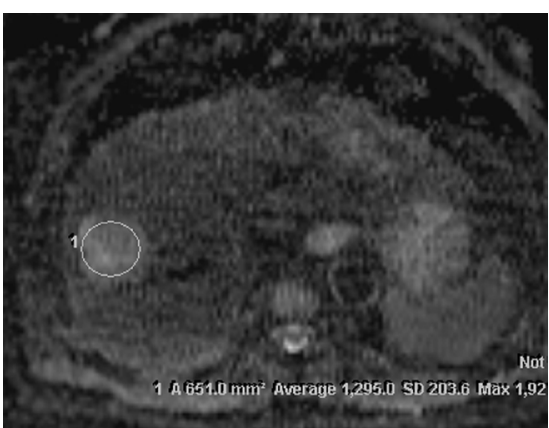

i

Fig. 2. Illustrations and representative diffusion-weighted images $\left(b=800 \mathrm{~s} / \mathrm{mm}^{2}\right)$ with correspond-ing apparent diffusion coefficient (ADC) maps of the three architectural types of hepatocellular carcinomas. Non-pseudoglandular (a-c), mixedpseudoglandular $(\mathrm{d}-\mathrm{f})$, and pure-pseudoglandular $(\mathrm{g}-\mathrm{i})$ types exhibit different $A D C$ values, as measured by the regions-ofinterest in the $A D C$ maps, depending on the size and number of pseudoglandular formations. 
The pd-HCCs $\left(1.04 \pm 0.16 \times 10^{-3} \mathrm{~mm}^{2} / \mathrm{s}\right)$ showed lower ADCs than the wd-HCCs $\left(1.10 \pm 0.15 \times 10^{-3} \mathrm{~mm}^{2} / \mathrm{s} ; \mathrm{P}=\right.$ $0.968)$ or $\mathrm{md}-\mathrm{HCCs}\left(1.14 \pm 0.21 \times 10^{-3} \mathrm{~mm}^{2} / \mathrm{s} ; \mathrm{P}=0.010\right)$ (Fig. 3b). Multivariate regression analysis with $A D C$ as the dependent variable, two independent variables of pseudoglandular formation and tumor differentiation showed statistical significance $(P=0.006$ and 0.030 , respectively). The $A D C$ values and pseudoglandular formation showed significant correlation only in the $\mathrm{md}-$ HCC subgroup ( $r=0.307, P=0.007)$, without any significant correlation in the wd-HCC $(r=0.105, P=0.600)$ and $p d-$ $\mathrm{HCC}(r=0.068, \mathrm{P}=0.685)$ subgroups.

\section{DISCUSSION}

According to the World Health Organization classification, HCCs are categorized into three different architectural categories: trabecular (plate-like), pseudoglandular (or

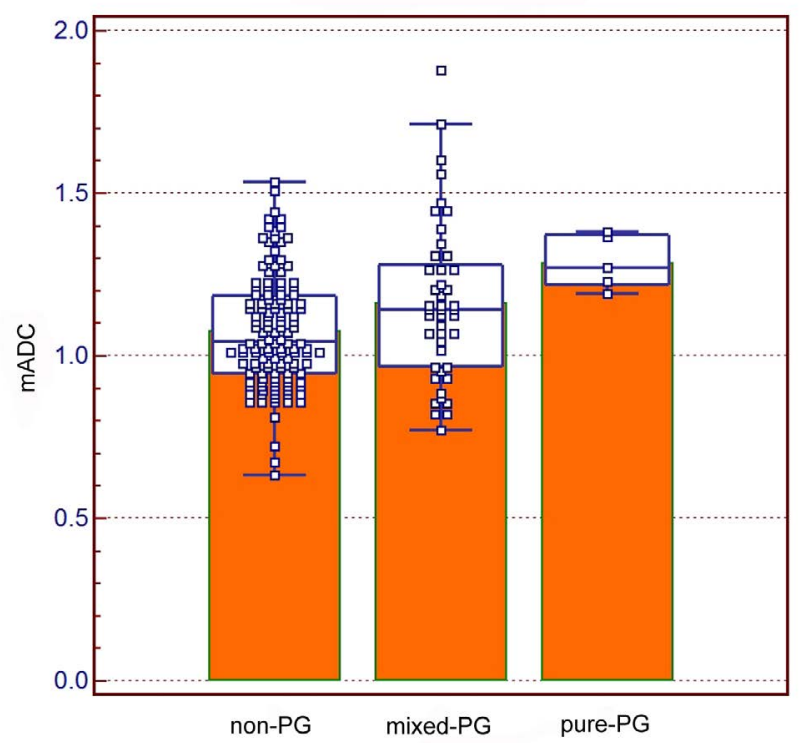

a

Fig. 3. Box-and-whisker plots showing the distribution, mean, and range of apparent diffusion coefficient (ADC) values for each (a) pseudoglandular type and (b) tumor grade. Individual data are represented as squares. The horizontal line in each box indicates the median value and the box indicates data from the $25^{\text {th }}$ to the $75^{\text {th }}$ percentile. The whiskers show the upper and lower extremes of data; outliers are marked as separate squares. (a) The mean ADC was significantly higher in pure pseudoglandular lesions $\left(n=5,1.29 \pm 0.08 \times 10^{-3} \mathrm{~mm}^{2} / \mathrm{s}\right)$ than in non-pseudoglandular lesions $(\mathrm{n}=132,1.08 \pm 0.17$ $\left.\times 10^{-3} \mathrm{~mm}^{2} / \mathrm{s} ; \mathrm{P}=0.003\right)$ or mixed-pseudoglandular lesions $\left(\mathrm{n}=45,1.16 \pm 0.24 \times 10^{-3} \mathrm{~mm}^{2} / \mathrm{s} ; \mathrm{P}=0.034\right)$. (b) The poorly differentiated hepatocellular carcinomas (HCCs) $\left(1.04 \pm 0.16 \times 10^{-3} \mathrm{~mm}^{2} / \mathrm{s}\right)$ exhibited a lower mean ADC than the welldifferentiated HCCs $\left(1.10 \pm 0.15 \times 10^{-3} \mathrm{~mm}^{2} / \mathrm{s} ; \mathrm{P}=0.968\right)$ or moderately differentiated HCCs $\left(1.14 \pm 0.21 \times 10^{-3} \mathrm{~mm}^{2} / \mathrm{s} ; \mathrm{P}=\right.$ 0.010). $\mathrm{mADC}=$ mean $A D C$ of two observers $\left(\times 10^{-3} \mathrm{~mm}^{2} / \mathrm{s}\right), \mathrm{md}=$ moderately differentiated, $\mathrm{pd}=$ poorly differentiated, $P G=$ pseudoglandular, $w d=$ well differentiated acinar), and compact patterns (20). The most common trabecular pattern shows cord-like tumor growth with variable thickness, depending on the tumor grade, separated by tumoral sinusoids with varying degree of dilatation. The pseudoglandular pattern comprises mostly a single layer of tumor cells with modified bile canaliculi and varying degrees of dilatation. They are usually admixed with the trabecular pattern, although a few pseudoglands may occasionally be formed by degeneration of thick trabeculae. The compact pattern rather commonly found in pd-HCCs contains inconspicuous tumoral sinusoids.

DWI measures the movement of water as a signal drop, which is partially affected by the distance that each water molecule moves in the soft tissue lesions. The diffusion coefficient reflects diffusion of extracellular water during clinical MRI. Several investigators have reported that HCCs with higher tumor grades exhibit lower ADCs than HCCs with lower tumor grades (9-16). Although the ADCs of the wd-HCCs appeared to be variable among the studies, the

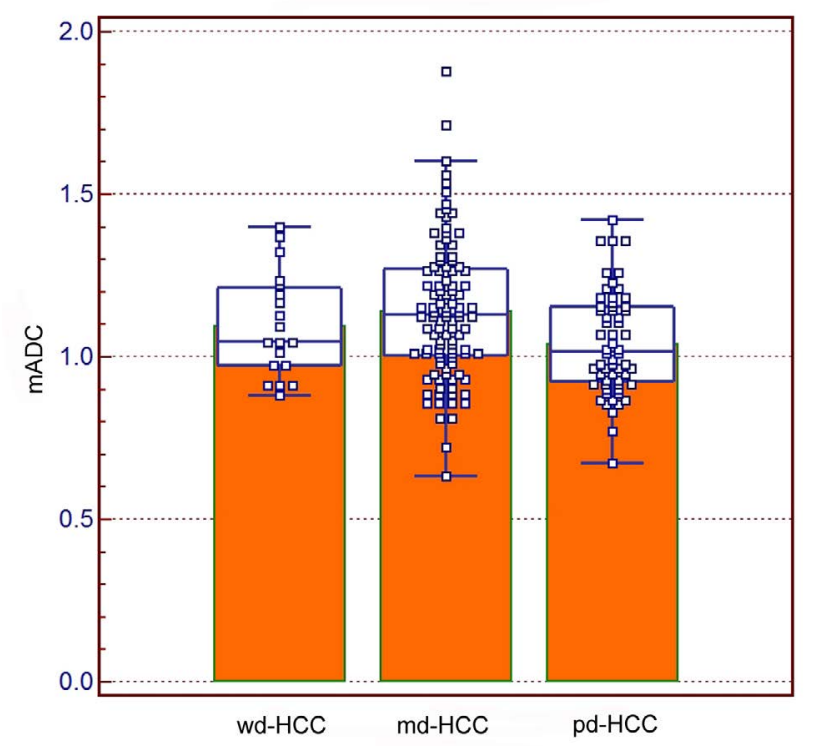

b 
pd-HCCs consistently exhibited lower ADCs in all published reports, suggesting that smaller tumor cells with thicker cellular plates and narrower sinusoidal spaces limit the water diffusion that occurs with de-differentiation in most of the tumors with trabecular architecture.

For HCCs with a pseudoglandular pattern, as in other structures or glandular lesions $(17,18)$, the glandular structure enhances the extracellular molecular motion to varying degrees, depending on the size and number of the pseudoglands, while other subtypes of compact cellularity negatively affect the molecular diffusion. The distribution ratio of the pseudoglandular formation in HCC is variable, and pure pseudoglandular HCC is quite uncommon $(<5 \%)$. In the present study, ADC values of pure-PG type were significantly higher than $A D C$ values of mixed-PG or non$P G$ types. The mixed-PG type yielded ADC values between pure-PG type and non-PG type. These results are consistent with our hypothesis that increased glandular formation is correlated with reduced restriction of water molecule motion and increased ADC values.

In the subgroup analysis according to the tumor grades, the present study revealed that pseudoglandular formation significantly affected ADCs in md-HCCs, but not in wdor pd-HCCs. Histologically, pseudoglandular pattern is a hall-mark of HCCs with lower degrees of cellular differentiation; the size of the pseudoglands in wd-HCCs tends to be smaller than in md-HCCs (20). The number of pseudoglandular patterns (pure-PG, 1; mixed-PG, 2; non-PG, 16) was too small for statistical validation. We suspected that glandular formation in wd-HCCs might not be adequate to affect the ADCs in the present study. In contrast, the extent of pseudoglandular formation might be inherently sparse in the pd-HCCs, which consist of dedifferentiated smaller tumor cells with thick tumor cell plates and narrow sinusoids. Regardless of the pathological description for the partial presence of glandular structures in this limited number of the patients, it would be difficult to change the constantly low ADCs of pd-HCCs, which coincided with all previous reports (9-16).

Our study has a few limitations. First, although only surgically resected and pathologically confirmed HCCs were included, pseudoglandular formation was not quantified and was roughly classified into three groups (pure-, mixed-, non-PGs), according to the descriptions in the pathology reports. Furthermore, the number of HCCs with pseudoglandular formation was too small to avoid selection bias, particularly in wd-HCCs. Ideally, retrospective analysis of the number and the size of pseudoglands using a standardized protocol by an experienced pathologist minimizes the bias in patient selection and provides a correlation coefficient relating the glandular formation and ADC. Second, we only investigated pseudoglandular patterns, without considering other architectural patterns (i.e., trabecular and compact). Trabecular type was the most common cellular type of HCC, and affected baseline ADCs depending on the histopathological tumor grades. The number of HCCs showing compact patterns was too small to be analyzed as an independent factor. Furthermore, the ADCs of compact patterns were expected to be restricted in the direction of cellular de-differentiation, which might be masked in the present study design. In the future, we hope to analyze each histopathological cellular type in a larger population. Third, aside from the histological tumor grades and architectural pattern, other determinants, including vascular perfusion, internal fibrosis, and other degenerative processes (e.g., hemorrhage or necrosis) may exist. For the perfusion fraction, intravoxel incoherent motion (IVIM) imaging quantifies the vascular perfusion and facilitates the separation of pure diffusion fraction from ADC (21-23). However, IVIM was not included in the routine hepatic MRI protocol in our institution and was, therefore, not available for analysis. In the area of degeneration, we avoided nonsolid tumor components during the localization of the ROI in each tumor, as in the previous studies (9-16); we believe that such influence was minimized in the present study.

In conclusion, our study suggests that the architectural variant of pseudoglandular formation could increase the ADCs of HCCs, especially in the md-HCCs. The pd-HCCs showed consistently lower ADCs, regardless of the presence of pseudoglandular formation. Despite several limitations, the results of the present study partially explain the variability of ADCs in clinical practice. Quantitative analysis of the size and extent of pseudoglandular formation in HCCs may represent a viable option for further studies verifying the present study findings.

\section{REFERENCES}

1. Rampone B, Schiavone B, Martino A, Viviano C, Confuorto G. Current management strategy of hepatocellular carcinoma. World J Gastroenterol 2009;15:3210-3216

2. Torre LA, Bray F, Siegel RL, Ferlay J, Lortet-Tieulent J, Jemal A. Global cancer statistics, 2012. CA Cancer J Clin 2015;65:87-108 
3. Jensen JH, Helpern JA, Ramani $A$, Lu H, Kaczynski K. Diffusional kurtosis imaging: the quantification of nongaussian water diffusion by means of magnetic resonance imaging. Magn Reson Med 2005;53:1432-1440

4. Kele $P G$, van der Jagt EJ. Diffusion weighted imaging in the liver. World J Gastroenterol 2010;16:1567-1576

5. Parikh T, Drew SJ, Lee VS, et al. Focal liver lesion detection and characterization with diffusion-weighted MR imaging: comparison with standard breath-hold T2-weighted imaging. Radiology 2008;246:812-822

6. Lowenthal D, Zeile $M$, Lim WY, et al. Detection and characterisation of focal liver lesions in colorectal carcinoma patients: comparison of diffusion-weighted and Gd-EOB-DTPA enhanced MR imaging. Eur Radiol $2011 ; 21: 832-840$

7. Lee MH, Kim SH, Park MJ, Park CK, Rhim H. Gadoxetic acid-enhanced hepatobiliary phase MRI and high-bvalue diffusion-weighted imaging to distinguish welldifferentiated hepatocellular carcinomas from benign nodules in patients with chronic liver disease. AJR Am J Roentgenol 2011;197:W868-875

8. Inchingolo $R$, De Gaetano AM, Curione $D$, et al. Role of diffusion-weighted imaging, apparent diffusion coefficient and correlation with hepatobiliary phase findings in the differentiation of hepatocellular carcinoma from dysplastic nodules in cirrhotic liver. Eur Radiol 2015;25:1087-1096

9. Muhi A, Ichikawa T, Motosugi U, et al. High-b-value diffusion-weighted MR imaging of hepatocellular lesions: estimation of grade of malignancy of hepatocellular carcinoma. J Magn Reson Imaging 2009;30:1005-1011

10. Heo SH, Jeong YY, Shin SS, et al. Apparent diffusion coefficient value of diffusion-weighted imaging for hepatocellular carcinoma: correlation with the histologic differentiation and the expression of vascular endothelial growth factor. Korean J Radiol 2010;11:295-303

11. Nishie A, Tajima T, Asayama $Y$, et al. Diagnostic performance of apparent diffusion coefficient for predicting histological grade of hepatocellular carcinoma. Eur J Radiol 2011;80:e29-33

12. Chang WC, Chen RC, Chou CT, et al. Histological grade of hepatocellular carcinoma correlates with arterial enhancement on gadoxetic acid-enhanced and diffusionweighted MR images. Abdom Imaging 2014;39:1202-1212

13. Chen J, Wu M, Liu R, Li S, Gao R, Song B. Preoperative evaluation of the histological grade of hepatocellular carcinoma with diffusion-weighted imaging: a metaanalysis. PLoS One 2015;10:e0117661

14. Guo W, Zhao S, Yang Y, Shao G. Histological grade of hepatocellular carcinoma predicted by quantitative diffusion-weighted imaging. Int J Clin Exp Med 2015;8:4164-4169

15. Tang $Y$, Wang $H, M a L$, et al. Diffusion-weighted imaging of hepatocellular carcinomas: a retrospective analysis of correlation between apparent diffusion coefficients and histological grade. Abdom Radiol (NY) 2016;41:1539-1545

16. Li X, Zhang K, Shi Y, Wang F, Meng X. Correlations between the minimum and mean apparent diffusion coefficient values of hepatocellular carcinoma and tumor grade. J Magn Reson Imaging 2016;44:1442-1447

17. Noworolski SM, Vigneron DB, Chen AP, Kurhanewicz J. Dynamic contrast-enhanced MRI and MR diffusion imaging to distinguish between glandular and stromal prostatic tissues. Magn Reson Imaging 2008;26:1071-1080

18. Wang $Y$, Chen ZE, Nikolaidis $P$, et al. Diffusionweighted magnetic resonance imaging of pancreatic adenocarcinomas: association with histopathology and tumor grade. J Magn Reson Imaging 2011;33:136-142

19. Yu JS, Kim JH, Chung JJ, Kim KW. Added value of diffusion-weighted imaging in the MRI assessment of perilesional tumor recurrence after chemoembolization of hepatocellular carcinomas. J Magn Reson Imaging 2009;30:153-160

20. Theise ND, Park YN, Curado MP, et al. Hepatocellular carcinoma. In Bosman FT, Carneiro F, Hruban RH, eds. WHO classification of tumours of the digestive system. 4th ed. Lyon: International Agency for Research on Cancer, 2010:205-216

21. Woo S, Lee JM, Yoon JH, Joo I, Han JK, Choi BI. Intravoxel incoherent motion diffusion-weighted MR imaging of hepatocellular carcinoma: correlation with enhancement degree and histologic grade. Radiology 2014;270:758-767

22. Granata V, Fusco R, Catalano O, et al. Intravoxel incoherent motion (IVIM) in diffusion-weighted imaging (DWI) for hepatocellular carcinoma: correlation with histologic grade. Oncotarget 2016;7:79357-79364

23. Shan 0 , Chen J, Zhang T, et al. Evaluating histologic differentiation of hepatitis B virus-related hepatocellular carcinoma using intravoxel incoherent motion and AFP levels alone and in combination. Abdom Radiol (NY) 2017;42:2079-2088 